\title{
Exploration of the Core Elements of Midrange Women's Clothing Store Display Based on Consumer Evaluation
}

\author{
Jingjing Nie ${ }^{1}$, Jing Zhou ${ }^{1}$, Hai Liu ${ }^{1} \&$ Jinhua Ruan ${ }^{1}$ \\ ${ }^{1}$ College of fashion, Shanghai University of Engineering Science, China \\ Correspondence: Jingjing Nie, College of fashion, Shanghai University of Engineering Science, China. E-mail: \\ 1649820376@qq.com
}

Received: April 17, 2015

Accepted: April 24, 2015

Online Published: April 27, 2015

doi:10.5430/jbar.v4n1p38

URL: http://dx.doi.org/10.5430/jbar.v4n1p38

\begin{abstract}
In women's apparel market, store displays are playing an increasingly important role. however, in midrange women's clothing store, which occupies the dominant position of the domestic women's market, display shows the characteristics of blindness and fuzziness and the core elements of the display is not clear and lack of business agility as well as consumer relevance, leading to no obvious effect. This article, through literature review, expert consultation, field investigation and questionnaire survey, using exploratory factor analysis, sums up the core elements of the midrange women's clothing store display, in order to provide positive guidance for the display planning.
\end{abstract}

Keywords: Consumer, Midrange Women's Clothing, Store Display

\section{Introduction}

In women's apparel market, female consumption concept is gradually changing, self-concept of consumption enhanced and consumer experience requirements increased, and store display has become one of the key factors that affects women's consumption. To some extent, it reflects the clothing enterprise positioning properties on the products, price, etc, and plays an important role in building the core value of brand and customer loyalty as well as wining the market core competitiveness, and has been more and more important for enterprises.

However, according to the current status of the display, especially, many domestic mid-range women's clothing store displays are blind and can not play the key role of core display elements, lack of commercial flexibility and consumer relevance, leading to no obvious effect. In professional research, the study is only one aspect of the display elements, ignoring the relationship between the elements as well as the quantitative analysis of elements. The research conclusions of quantitative analysis of clothing display elements are few.

The article, through the combination of qualitative and quantitative methods, explores the importance of the various elements of the midrange women's clothing store display and summarizes the core elements, so as to provide positive guidance for the fashion display planning.

\section{Current Research}

Since the $1980 \mathrm{~s}$, accompanied by the changes of marketing concept, clothing store display gradually developed into a kind of visual marketing strategy method, along with the emergence of a number of related works and studies at home and abroad.

Tony Morgan edited "Visual merchandising: window and in-store displays for retail", and carried on the detailed explanation from the history of visual merchandising, visual merchandising duties, store design, window display, store displays, use of models racks, visual display division studio and other aspects; Fang Zeming, in "Research on the problem about domestic apparel brands in the visual marketing", pointed out slow updates, not enough emphasis on the application of color, lack of overall planning, management confusion and other issues existing in the clothing store display and gave the corresponding countermeasures; Li Na studied what psychological effects of the consumers in costume exhibition space in the article of "costume exhibition space constitute research and consumer psychology effect", and explained the reason from a psychological point of view; Chang Sang, in "Mix of colors and fabrics in clothing display design", detailed the collocation of color and fabric as well as various factors affecting the actual operation; Qi Linlin discussed the importance of window design, techniques, ideas, prospects and other 
aspects in the article of "Talking clothing display in window design".

Although some tests and research on apparel visual merchandising have been done, most are sensual analysis, and the studies are only one aspect of the display elements, lack of quantitative research on the importance of various elements of the display, and there is no discuss about the core elements of midrange women's clothing store display, so no adequate guidance to the overall planning of store display

\section{Women's Clothing Store Display}

Women's clothing store display is a visual expression, and it refers to a kind of performance technology to show the characteristics of the women's apparel, using a variety of props (including lighting, display, background music, etc.), combined with product positioning, brand culture and other different techniques. The exhibition need meet women's brand positioning and design, reflect the lifestyle and popular features that the clothes aims to show, and vary accompanying with different purposes of the display.

Reasonable women's clothing store display will show the design characteristics to consumers in the most effective way, and this show sometimes replaces parts of store staff's clothing introduction, and enhance the consumers' clothing understanding in the most direct way, which provides consumers with a rich visual experience and then stimulates the desire to buy.

Besides, store display, as an effective means of visual marketing, can not only deepen the consumer impression of clothing products directly, giving consumers a good experience as well as stimulating the purchase desire, but also promote good clothing brand image on a higher level, establishing a corporate image.

\section{Elements of Women's Clothing Store Display}

Because of the diversity of women's clothing store display, its display elements are rich and various, we can preliminarily understand the elements from three aspects, that is display image, display technology and display equipment.

First, about display image, the elements associated with the image of the store are lights, music, windows, smell, decoration, color, light boxes, and staff dress, and the elements associated with the propaganda image are price tag, brand label, promotional brochure, product manuals, posters, bags, etc.

Furthermore, as to display technology, elements associated with the display mode are integrated display, focus display, repeat display, partition display, horizontal display, hanging on display, side hung on display, etc., and line planning-related elements are sight, channel, space allocation, dressing room settings, etc.

Finally, the elements associated with display equipment are racks, shelves, containers, models, display stands, etc.

\section{Display Elements Research Based on Consumer Evaluation}

\subsection{Research Methods}

This article, through the relevant literature, secondary data, relevant professional consulting and sells field investigation, collects the various elements of store display, and after analysis, deliberation as well as arrangement, extracts elements for a total of 26 covering all dimensions, then designs the questionnaire and conducts exploratory factor analysis on sample data by SPSS 19.0. The purpose of the study is to clear the importance of display elements of midrange women's clothing store in the process that female consumers walk into the store, choice the clothing and finally purchase it.

The questionnaires are mainly sent out in major shopping malls, stores and other crowd numerous locations, and also are issued from the network. A total of 200 questionnaires were sent out, and 181 valid questionnaires returned, so the effective rate is $82.27 \%$.. The main survey location is Songjiang district in Shanghai, using the questionnaire for scoring system

\subsection{Exploratory Factor Analysis Results}

The study, by calculating the internal consistency coefficient, gets $\alpha$ coefficients of the questionnaire of 0.733 , indicating the questionnaire has a higher reliability, and. gets correlation index KMO results of the variables for $0.706>0.7$ via correlation test, indicating little difference in the degree of correlation between variables, and gets ball hypothesis test' significance level $\mathrm{Sig}<0.05$ about independent variables, showing variable independence test is significant. That's to say, the variables are not independent and suitable for factor analysis.

This article takes the method of principal component analysis to extract factors from 26 display elements, and the number of factors to be extracted is determined by charts of communalities and total variance explained. Finally, we 
determine the factor classification of each original element by rotated component matrix.

First, in the communalities table, the variables' communalities are all above 0.5 , so we can extract common factor from all 26 display element variables.

Next, determine the number of common factors to explain the total variables by total variance explained table. (see table 1)

Table 1. Total Variance Explained

\begin{tabular}{|c|c|c|c|c|c|c|c|c|c|}
\hline \multirow{3}{*}{ Compo-nent } & \multicolumn{3}{|c|}{ Initial Eigenvalues } & \multicolumn{3}{|c|}{$\begin{array}{l}\text { Extraction Sums of Squared } \\
\text { Loadings }\end{array}$} & \multicolumn{3}{|c|}{$\begin{array}{l}\text { Rotation Sums of Squared } \\
\text { Loadings }\end{array}$} \\
\hline & & $\%$ of & & & $\%$ of & & & $\%$ of & \\
\hline & Total & Variance & Cumulative & oTotal & Variance & Cumulative & oTotal & Variance & Cumulative $\%$ \\
\hline 1 & 4.960 & 19.840 & 19.840 & 4.960 & 19.840 & 19.840 & 3.884 & 15.535 & 15.535 \\
\hline 2 & 3.919 & 15.677 & 35.517 & 3.919 & 15.677 & 35.517 & 3.667 & 14.669 & 30.204 \\
\hline 3 & 3.120 & 12.478 & 47.995 & 3.120 & 12.478 & 47.995 & 3.385 & 13.541 & 43.745 \\
\hline 4 & 2.268 & 9.072 & 57.067 & 2.268 & 9.072 & 57.067 & 2.727 & 10.909 & 54.654 \\
\hline 5 & 2.075 & 8.299 & 65.366 & 2.075 & 8.299 & 65.366 & 2.678 & 10.712 & 65.366 \\
\hline 6 & .971 & 3.885 & 69.251 & & & & & & \\
\hline 7 & .902 & 3.608 & 72.859 & & & & & & \\
\hline 8 & .804 & 3.216 & 76.076 & & & & & & \\
\hline 9 & .723 & 2.892 & 78.967 & & & & & & \\
\hline 10 & .682 & 2.726 & 81.693 & & & & & & \\
\hline
\end{tabular}

From the above table1, we can get five important factors from selecting factors whose eigenvalues are greater than one with the cumulative variance contribution rate of $65.366 \%$. The five common factors can explain more than $65 \%$ of the total variance, including the most of the original variable information.

Then, we can determine the main component of the common factors, classify the variables and name factors by rotated component matrixa table.(see table2) 
Table 2. Rotated Component Matrixa

\begin{tabular}{|c|c|c|c|c|c|}
\hline & \multicolumn{5}{|c|}{ Component } \\
\hline & 1 & 2 & 3 & 4 & 5 \\
\hline Store view & .005 & .765 & .002 & .007 & -.053 \\
\hline $\begin{array}{l}\text { The center part of wall ,important } \\
\text { shelves or exhibition center }\end{array}$ & -.024 & .801 & -.179 & .099 & .042 \\
\hline Window display & -.103 & .840 & .088 & .021 & .007 \\
\hline Interior space of the store & .063 & .797 & .007 & .047 & -.055 \\
\hline Accentl ighting & .110 & .094 & .824 & -.006 & -.055 \\
\hline Brochures & .299 & -.062 & .136 & -.001 & .714 \\
\hline Mood lighting & .028 & -.090 & .793 & -.049 & .083 \\
\hline Basic lighting & .054 & .031 & .836 & -.129 & -.131 \\
\hline The primary and secondary of color & .773 & -.038 & -.082 & -.116 & .139 \\
\hline Shop air & .079 & -.070 & .779 & .069 & -.021 \\
\hline Store channel & -.075 & .745 & -.107 & .063 & .231 \\
\hline Popular color & .834 & .039 & .008 & -.097 & .032 \\
\hline Gradient colors & .769 & -.018 & .102 & -.041 & .254 \\
\hline Contrasting colors & .697 & .094 & .128 & -.087 & .044 \\
\hline Fitting room's placement & .385 & .670 & .058 & -.161 & .128 \\
\hline Integrated display & .019 & -.099 & .033 & .853 & .095 \\
\hline Decorative colors & .749 & -.010 & .113 & -.056 & -.034 \\
\hline Similar color matching & .716 & -.015 & -.051 & -.084 & .179 \\
\hline Focal display & -.295 & .110 & -.050 & .770 & -.162 \\
\hline Background music & -.067 & -.077 & .774 & -.183 & .102 \\
\hline Respective display & -.045 & .093 & -.197 & .827 & -.095 \\
\hline POP advertising & .106 & .050 & -.045 & -.082 & .836 \\
\hline The logo & .121 & .113 & -.022 & -.061 & .812 \\
\hline Repeated display & -.190 & .043 & -.066 & .755 & .012 \\
\hline Price tag & .035 & .061 & -.050 & .030 & .728 \\
\hline
\end{tabular}

From the perspective of the data of table 2, factor 1 is mainly composed of decorative colors, contrasting colors, similar color matching, gradient colors, the primary and secondary of color and popular color 6 elements, named it color factor.

Factor 2 is mainly composed of store view, the center part of wall, important shelves or exhibition center, interior space of the store, fitting room's placement, window display and store channel 7 elements, named it spatial layout factor.

Factor 3 is mainly composed of basic lighting, accent lighting, mood lighting, background music and shop air 5 elements, named it environment factor.

Factor 4 is mainly composed of integrated display, focal display, respective display and repeated display 4 elements, named it display mode factor. 
Factor 5 is mainly composed of pop advertising, the logo, price tag and brochures 4 elements, named it brand promotion factor.

\subsection{Analysis of the Relative Importance of Display Elements}

From the factor analysis results, we can sum up index system of women's clothing display elements and the relative importance of display elements. The following is the index system of women's clothing display elements.(see figure 1)

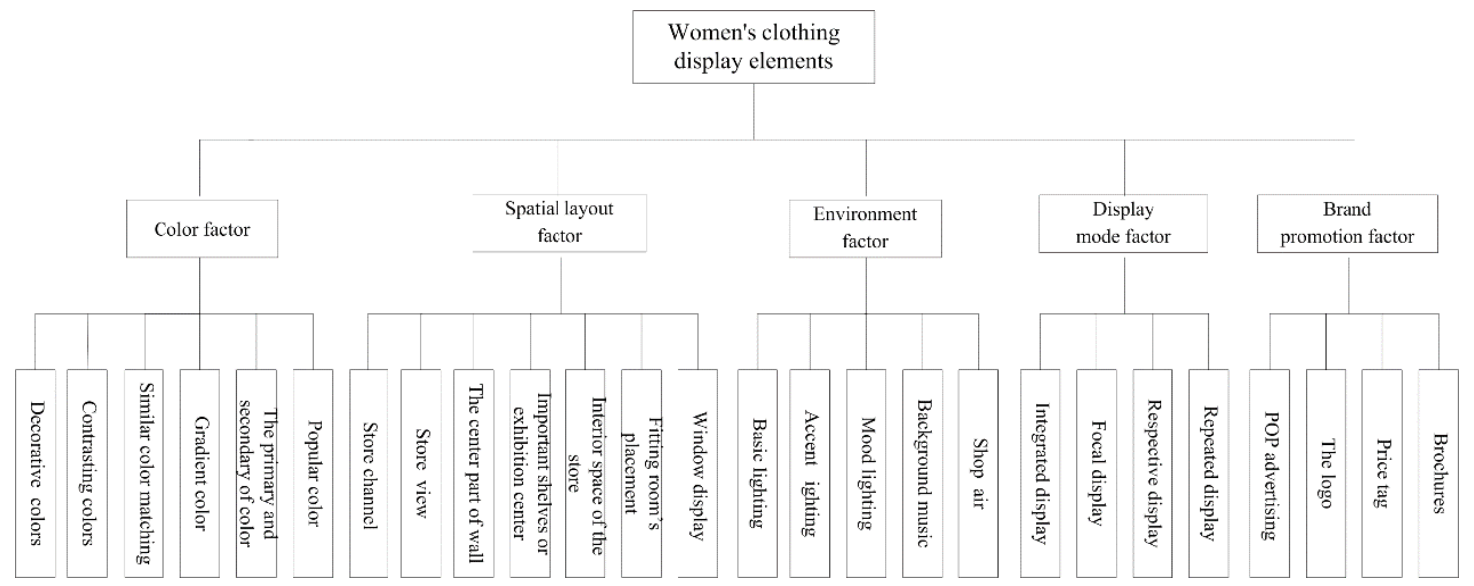

Figure 1: The Index System of Women's Clothing Display Elements

In the factor analysis, factor loading value of each variable represents the variable contribution to the common factor, the greater the factor loading value is, the larger the variable contribution to the common factor is,namely, the variables' relative importance to common factors is larger.

From the table 2, the variables of popular color and the primary and secondary of color are more important to $\mathrm{F} 1$,which indicates that in terms of color elements, it should pay particular attention to the selection and matching of quarterly popular color, as well as the primary and secondary of various clothing so as to arouse consumers' attention and interest to attract them into the stores.

For F2, in addition that the window display has a relatively important role, the center part of wall ,important shelves or exhibition center also have massive significance, which shows that it should not only stress the window design but also the setting of the center part of wall, important shelves or exhibition center. Besides, , the store view also has an important influence, indicating that store display should give full consideration to the convenience of consumers' sights to allow them to find their favorite clothing as soon as possible and have a clear resolution to the clothing.

Basic lighting and accent lighting play a more important position in F3, indicating that the cognitive evaluation of the mid-range women's clothing consumers is more influenced by basic lighting and accent lighting ,compared with light, air, background music and other environment factors. Basic lighting refers to the lighting that provides adequate basic brightness in the store, and accent lighting is a lighting technique that is specially used to emphasize or highlight colors styles and design details, etc. Therefore, special attention should be paid to the collocation of basic lighting and accent lighting in order to guide consumers to browse and select clothes in the stores and attract them to the clothing.

The variables of integrated display and respective display are more important to F4, indicating that the target consumer group is more interested in the display method of classification of clothing show and clothing combinations show.

POP advertising and the logo are more important to F5, which shows that in the aspects of branding, consumers pay more attention to the impact of logo and pop advertising, in addition, they also have some concerns about the price tag.

\section{Store Display Planning Proposals}

Midrange women's clothing is a brand product that has mass-consumer groups with mass production, and its brand image design is an imitation of the senior brand's. The quality of the material and workmanship is slightly worse than the senior fashion and it just provides general product quality and service consumers need. "Beauty, novelty, and economization " is consumer psychology demand of midrange women's clothing. Therefore, the clothing design 
is novel, short fashion cycles, fast style or color changes as well as high timeliness, etc. And its target consumer groups are the working-class urban and wealthy families in rural. On the basis of the market consumption characteristics of mid-range women's clothing combining with the relevant data of the study, we can put forward the following recommendations in three aspects of store display.

First, in the aspect of store atmosphere and clothing culture, in view of the target consumer group of midrange women's clothing have an important evaluation on basic lighting, accent lighting and pop advertising, about all the elements in the atmosphere and the brand promotion, we should focus on the design and setting of POP advertising and lights. However, the same display design with different lights use will have completely different influence on consumer psychology, and its expression of clothing is completely different, and good pop advertising will have the effect of the wasted effort in promoting the culture. Therefore, special attention should be paid to the appropriateness of light and pop advertising design, and at the same time, take into account shop air, background music, brochures and other factors, in order to create a theme environment to meet consumer's mind to form a good impression in the minds of customers.

Furthermore, in the aspect of the store space setting, taking into account consumers' high attention to window display and the center part of wall ,important shelves or exhibition center as well as the novelty, setting in space should focus on these two points, with the remaining elements cooperating, such as store view, interior space of the store and store channel ,to defuse the conflicts between limited display space and various types of clothing. Through this strategy, the various parts of the store display can be coordinated to form an effective market connected with sales, consumers can see more of their favorite clothing, resulting in attention, interest and association, and thus contribute to consumption.

Finally, in the aspects of clothing display, special attention should be paid to the color matching and display modes. Color is a means of communication spreading faster than words. It is not only able to form a pleasant environment for consumers, but also to provide a beautiful visual experience for the purchaser. Sales of women's clothing at the same time are also the color sale, and appropriate display ways give customers a good experience in "easy viewing, easy touch and easy to choose". Combined with midrange women's clothing' features of multi-color and popular fast-changing, under the guidance of the research results, emphasis should be placed in the arrangement of popular colors and colors matching, as well as selection of the method of integrated display and respective display, combining other elements in color and display mode, in order to show the characteristics of various types of clothing.

\section{Conclusion}

This article, through the relevant literature, secondary data, relevant professional consulting and sells field investigation, collects store display elements for a total of 26 covering all dimensions, and summarizes them into 5 common factors after questionnaires and exploratory factor analysis, that is color factor, spatial layout factor ,environment factor, display mode factor and brand promotion factor., and analyze the relative importance of each element inside the common factors. Among them, the elements of popular color ,the primary and secondary of color, window display, the center part of wall ,important shelves or exhibition center, integrated display ,respective display, basic lighting and accent lighting and POP advertising are in a relatively important position in common factors, which are the core elements that should be given the primary consideration.

Because of the time, funds and other conditions, the main area of this research is the Shanghai Songjiang District, which may make conclusions with some limitations. In addition, since some elements of the clothing store display are difficult to describe with words, the questionnaire selected corresponding picture for reference, which may bring some interference to the objectivity of conclusion.

This paper studies the importance of various elements of midrange women's clothing store display and sums up the core elements, and has positive significance for the business to select and set the core elements, which will help companies develop a good marketing strategy. In the future, we can carry out in-depth research on the choice of display elements and visual merchandising strategy, developing marketing strategies based on the core elements to harvest the good marketing results.

\section{Acknowledgements}

This study was funded by Shanghai University of Engineering Science 2015 -year Innovation Fund for Graduate Students.

This study was funded by Cluster Brand Development Strategy Research of Shanghai "leading" Group, the funded project No.E1-0903-14-01172. 


\section{References}

Cao Chunyan. (2013). Brand positioning: to meet the needs of different levels of consumption in the women's show. Shaoguan University - Social $\quad$ Sciences, $\quad$ 2013,05: http://dx.chinadoi.cn/10.3969\%2fj.issn.1007-5348.2013.05.034.

Chang Sang, Zhuyue Zhong. (2014). Clothing display design with color and fabric. Apparel,2014,01: 73. http://dx.chinadoi.cn/10.3969\%2fj.issn.1673-0356.2014.01.023.

Fang Zeming. (2007). Research on the problem about domestic apparel brands in the visual marketing. Fujian Normal, 2007. http://dx.chinadoi.cn/10.7666/d.y1184403.

Li Na. (2011). Costume Exhibition space constitute research and consumer psychology effect. Dalian Polytechnic University,2011: 1-5.

Li Qin etc. (2014). Store visual merchandising. Beijing: Tsinghua University Press. Beijing Jiaotong University Press, 2014,56-88.

Liu Xiaohong, Liu Dong. (2008). Apparel marketing. Beijing: China Textile Press, 2008.142.

Liu Xiaohong. (2005). Apparel marketing data analysis. Beijing: China Textile Press, 2005,09: 247-251.

Sun Xiaodan. (2013). Casual store display of art to explore. Progress in Textile Science and Technology, 2013 (3) .88-89. http://dx.chinadoi.cn/10.3969\%2fj.issn.1673-0356.2013.03.027.

Tony Morgan. (2009). Visual merchandising: window and in-store displays for retail. Beijing: China Textile Press, 2009.3.

Zhang Xiaoli. (2010). Clothing display design. Beijing: Beijing Institute of Technology Press, 2010.02: 31-65. 